\title{
A numerical study of two-phase Stokes flow in an axisymmetric flow-focusing device
}

\author{
Jensen, Mads Jakob; Stone, H.A.; Bruus, Henrik
}

Published in:

Physics of Fluids

Link to article, DOI:

$10.1063 / 1.2214461$

Publication date:

2006

Document Version

Publisher's PDF, also known as Version of record

Link back to DTU Orbit

Citation (APA):

Jensen, M. J., Stone, H. A., \& Bruus, H. (2006). A numerical study of two-phase Stokes flow in an axisymmetric flow-focusing device. Physics of Fluids, 18(7), 077103. https://doi.org/10.1063/1.2214461

\section{General rights}

Copyright and moral rights for the publications made accessible in the public portal are retained by the authors and/or other copyright owners and it is a condition of accessing publications that users recognise and abide by the legal requirements associated with these rights.

- Users may download and print one copy of any publication from the public portal for the purpose of private study or research.

- You may not further distribute the material or use it for any profit-making activity or commercial gain

- You may freely distribute the URL identifying the publication in the public portal

If you believe that this document breaches copyright please contact us providing details, and we will remove access to the work immediately and investigate your claim. 


\title{
A numerical study of two-phase Stokes flow in an axisymmetric flow-focusing device
}

\author{
Mads Jakob Jensen \\ MIC - Department of Micro and Nanotechnology, DTU Bldg. 345 East, Technical University of Denmark, \\ DK-2800 Kongens Lyngby, Denmark \\ Howard A. Stone \\ Division of Engineering and Applied Sciences (DEAS), Harvard University, 308 Pierce Hall, Cambridge, \\ Massachusetts 02138 \\ Henrik Bruus \\ MIC - Department of Micro and Nanotechnology, DTU Bldg. 345 East, Technical University of Denmark, \\ DK-2800 Kongens Lyngby, Denmark
}

(Received 14 November 2005; accepted 24 May 2006; published online 6 July 2006)

\begin{abstract}
We present a numerical investigation of the time-dependent dynamics of the creation of gas bubbles in an axisymmetric flow-focusing device. The liquid motion is treated as a Stokes flow, and using a generic framework we implement a second-order time-integration scheme and a free-surface model in MATLAB, which interfaces with the finite-element software FEMLAB. We derive scaling laws for the volume of a created bubble and for the gas flow rate, and confirm them numerically. Our results are consistent with existing experimental results by Garstecki et al. [Phys. Rev. Lett. 94, 164501 (2005)], and predict a scaling yet to be observed: the bubble volume scales with the outlet channel radius to the power of 4 and the surface tension. Our axisymmetric simulations further show that the collapse of the gas thread before bubble snap-off is different from the recent experimental results. We suggest that this difference is caused by differences in geometry between experiments and the simulations. () 2006 American Institute of Physics. [DOI: 10.1063/1.2214461]
\end{abstract}

\section{INTRODUCTION}

Studying the generation of bubbles and drops on the micrometer scale in a predefined geometry makes it possible to investigate a variety of physical phenomena, such as capillarity, dripping, and bubbling processes. The initial work on this topic has been mainly experimental and often it has been driven by industrial applications of such devices; e.g., drop formation in ink-jet printing. ${ }^{1}$ To better understand experimental results, and possibly aid design of microfluidic devices for controlling multiphase flow, numerical modeling of drop and bubble formation is also necessary. Modeling the free-surface dynamics includes the use of direct numerical techniques such as the volume of fluid methods, tracer methods, and boundary-integral methods. ${ }^{2-5} \mathrm{~A}$ wide variety of analytical and semi-analytical models have also been introduced such as, for example, the thin jet approximation. ${ }^{6-10}$

In recent years, studies have been concerned with the so-called flow-focusing configuration. ${ }^{11-16}$ One implementation possible with microfluidic devices allows for the generation of controlled multiphase flows; this has mainly been investigated experimentally. Many interesting physical phenomena have been described, including flow-rate controlled breakup of gas threads, ${ }^{13}$ but also the appearance of chaotic behavior as reported in other bubbling devices. ${ }^{6,12,14}$

In this paper, which is inspired by the experimental work of Garstecki et al., ${ }^{12,13}$ we present a numerical study of a microfluidic flow-focusing device. The study is restricted to the Stokes-flow regime (low Reynolds number) in axisymmetric geometries. ${ }^{17}$ We derive a scaling law for the volume of created bubbles, which is in agreement with those observed experimentally. We also predict a new scaling: the bubble volume is linearly proportional to the liquid-gas surface tension an effect hitherto not observed in the experimentally realized geometry, to our knowledge. Finally, we observe compressibility of gas bubbles in our system. Nevertheless, it is important to note that we do not produce a direct verification of experimental results as our axisymmetric geometry is different from the planar one used in experiments.

To simulate the free-surface dynamics, we have implemented a second-order Runge-Kutta time algorithm in MATLAB ${ }^{18}$ coupled to the commercial finite-element program FEMLAB ${ }^{19}$ to solve the incompressible Stokes-flow equations for the velocity and pressure fields. This approach enables us to account for the full geometry of a device including walls, inlets, and free liquid-gas interfaces. At the location of a free surface, the normal and tangential stress conditions are enforced including the Young-Laplace pressure jump associated with a curved interface. We describe the governing equations and the numerical model in Sec. II, we discuss the results in Sec. III, and finally conclude in Sec. IV.

\section{THE MODEL AND NUMERICAL FORMULATION}

\section{A. The system}

The axisymmetric flow-focusing device is depicted schematically in Fig. 1. A gas thread (light gray) under constant pressure $p_{\text {gas }}$ is focused into an orifice (dark gray) by a liquid 


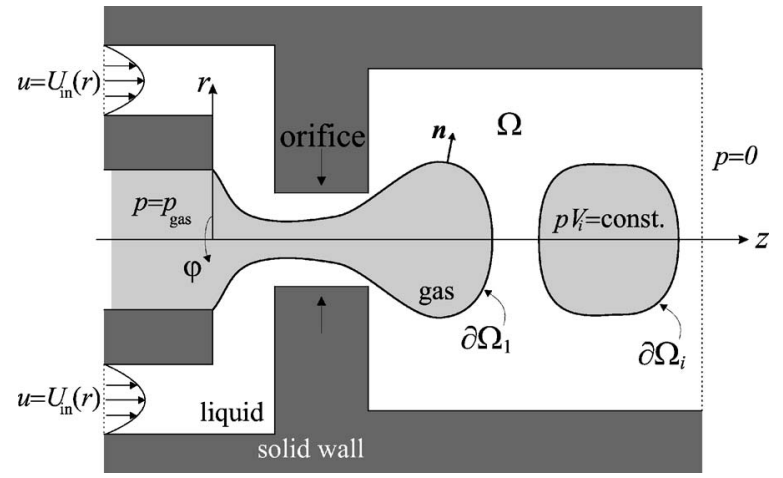

FIG. 1. Sketch of the axisymmetric flow-focusing device with liquid domain $\Omega$ (white), rigid boundaries (dark gray), gas bubbles (light gray), liquid-gas interfaces $\partial \Omega_{i}$, interface normal $\boldsymbol{n}$, pressure in the gas $p_{\text {gas }}$, and the inlet velocity $u=U_{\text {in }}(r)$ of the liquid. The azimuthal angle $\varphi$ is depicted to emphasize the axisymmetric geometry.

stream (white). It is natural to use cylindrical $(z, r, \varphi)$ coordinates, whereby the liquid flow-rate at the inlet is

$$
Q_{\text {in }}=\int_{\partial \Omega_{\text {in }}} \boldsymbol{u} \cdot \boldsymbol{n} d A=2 \pi \int_{\Delta r} U_{\text {in }}(r) r d r,
$$

where $\partial \Omega_{\text {in }}$ is the inlet boundary, $\boldsymbol{u}=U_{\text {in }}(r) \boldsymbol{e}_{z}$ is the flow profile at the inlet, $\boldsymbol{n}$ is the surface normal, and $\Delta r$ is the width of the inlet in the radial direction $r$. As the system is axisymmetric about the $z$ axis the liquid is focusing the gas thread uniformly from all sides; i.e., for all $\varphi \in[0,2 \pi[$. Over a large range of flow parameters, the gas thread breaks periodically and bubble snap-off occurs. The bubbles flow downstream into the outlet.

The sketch in Fig. 1 also shows the boundary conditions: (a) at the liquid inlet the fluid velocity $U_{\text {in }}(r)$ is specified [see Eq. (1)]; (b) at the outlet the pressure is set to zero; (c) on all solid walls we assume no slip $\boldsymbol{u}=\mathbf{0}$; and (d) at the liquid-gas interfaces $\partial \Omega_{i}$ the full stress condition is fulfilled, including the Young-Laplace pressure jump associated with a curved interface; and (e) at the line $r=0$ a symmetry condition is applied.

\section{B. Governing equations}

Compared to the liquid, the shear stress and the viscosity of the gas are negligible and the pressure variations are expected to be small, so that we only need to specify a pressure in the gas domains. Moreover, we assume that the gas is an isothermal ideal gas that does not exchange molecules with the surroundings. In the gas thread the pressure $p_{\text {gas }}$ is constant, and in the isolated $i$ th bubble that is formed, we set the pressure $p_{i}$ according to

$$
p_{i} V_{i}=p_{\text {gas }} V_{\text {snap }}=\text { const, }
$$

where $V_{i}$ is the volume of bubble $i$ and $V_{\text {snap }}$ is the volume of a bubble at snap-off.

In the present study, we assume that the liquid involved is Newtonian. Moreover, since the Reynolds number in the experiments falls in the range 0.02-0.2, we assume that the flow is in the Stokes limit. The flow field thus satisfies the equation

$$
\boldsymbol{\nabla} \cdot \boldsymbol{\sigma}=0
$$

where $\boldsymbol{\sigma}$ is the Cauchy stress tensor. A Newtonian fluid in an axisymmetric system is effectively a two-dimensional system and the four components of the stress tensor are

$$
\sigma_{i j}=\mu\left(\frac{\partial u_{i}}{\partial x_{j}}+\frac{\partial u_{j}}{\partial x_{i}}\right)-p \delta_{i j} .
$$

where $\left(x_{1}, x_{2}\right)=(z, r),\left(u_{1}, u_{2}\right)=(u, v)$ are the velocity components, $p$ is the pressure, and $\mu$ is the dynamic viscosity of the fluid. Apart from the momentum equation given in Eq. (3) and the constitutive stress tensor, the continuity equation is needed:

$$
\boldsymbol{\nabla} \cdot \boldsymbol{u}=0 .
$$

To make parametric studies of physical variables in a dimensionless setup, we introduce the dimensional length $d$ and the velocity $U$, the values of which will be discussed later. Using this, we now rescale position $\boldsymbol{x}$, velocity $\boldsymbol{u}$, time $t$, and pressure $p$ as

$$
\begin{aligned}
& \boldsymbol{x}=\tilde{\boldsymbol{x}} d, \\
& \boldsymbol{u}=\tilde{\boldsymbol{u}} U, \\
& t=\tilde{t} \frac{d}{U}, \\
& p=\tilde{p} \frac{\mu U}{d} .
\end{aligned}
$$

The tilde is used to identify nondimensional parameters $\tilde{\boldsymbol{x}}, \tilde{\boldsymbol{u}}$, $\tilde{t}$, and $\tilde{p}$. Introducing the rescaling Eq. (6) of the flow parameters, it is possible to reformulate Eqs. (3)-(5) into a divergence form. Using the axisymmetric form of the governing equations and some algebra yields

$$
\begin{gathered}
\boldsymbol{\nabla} \cdot\left[\begin{array}{cc}
\tilde{r}\left(2 \frac{\partial \tilde{u}}{\partial \widetilde{z}}-\tilde{p}\right) & \tilde{r}\left(\frac{\partial \tilde{u}}{\partial \widetilde{r}}+\frac{\partial \tilde{v}}{\partial \widetilde{z}}\right) \\
\tilde{r}\left(\frac{\partial \tilde{u}}{\partial \tilde{r}}+\frac{\partial \tilde{v}}{\partial \widetilde{z}}\right) & \tilde{r}\left(2 \frac{\partial \tilde{v}}{\partial \tilde{r}}-\tilde{p}\right) \\
0 & 0
\end{array}\right] \\
=\left[\begin{array}{cc}
0 \\
-\tilde{p}+2 \frac{\tilde{v}}{\tilde{r}} \\
\tilde{v}+\tilde{r}\left(\frac{\partial \tilde{v}}{\partial \tilde{r}}+\frac{\partial \tilde{u}}{\partial \widetilde{z}}\right)
\end{array}\right],
\end{gathered}
$$

where $\boldsymbol{\nabla}=(\partial / \partial \widetilde{z}, \partial / \partial \widetilde{r})$. The first two rows in the system represent the momentum equations and the third row is the incompressibility condition. Note that the matrix denoted $\boldsymbol{\Gamma}$ at the left of the equal sign contains $\widetilde{r}$ times the Cauchy stress tensor. Rewriting the governing equations into the somewhat unusual form of Eq. (7) is beneficial for the numerical implementation of the governing equations into FEMLAB, as discussed in the next section. 


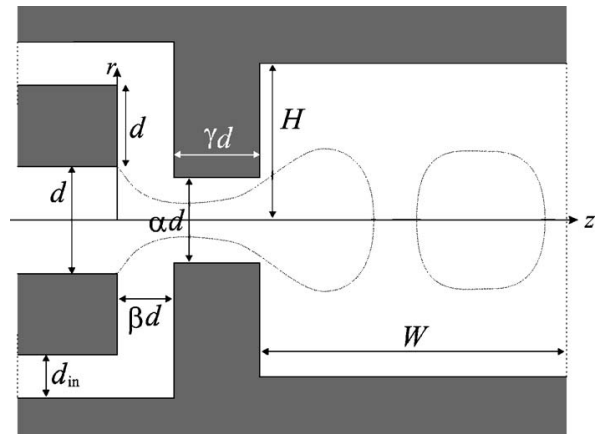

FIG. 2. Sketch of the axisymmetric flow-focusing device with all relevant lengths shown. Most important are the gas inlet diameter $d$, the orifice diameter $\alpha d$, the distance from the orifice to the gas inlet $\beta d$, and the length of the orifice $\gamma d$.

At the location of the free interface $\partial \Omega_{i}$ (see Fig. 1) the stress is continuous in the tangential direction, but exhibits a discontinuity in the normal direction $\boldsymbol{n}$ given by the YoungLaplace pressure. Thus, at the free interface the stress condition is

$$
\boldsymbol{n} \cdot \boldsymbol{\sigma}=\left(2 \gamma_{\mathrm{lg}} \kappa-p_{\text {gas }}\right) \boldsymbol{n},
$$

where $\gamma_{\lg }$ is the surface tension of the liquid-gas interface, and $\kappa$ is the mean curvature of the surface. At the free surface, the interface must move at the speed of a fluid particle just next to it, which is the kinematic boundary condition

$$
\frac{d x}{d t}=\left.\boldsymbol{u}(\boldsymbol{x})\right|_{x \in \partial \Omega_{i}},
$$

where we take the total time derivative.

In the Stokes-flow limit, the time-dependent problem reduces to a quasistationary problem in the following sense. Given an interface shape, we solve for the velocity field $\boldsymbol{u}$ and the stress field $\boldsymbol{\sigma}$ in the liquid. If the stress including pressure is nonzero at any point on the liquid-gas interface, this interface will move. Mathematically, the time dependence only enters through the kinematic boundary condition Eq. (9) at this free surface. The flow is thus implicitly a function of the interface shape. Although we are in the Re $=0$ limit, nonlinearity is nevertheless introduced to the dynamics of the problem by the curvature of the free interface appearing in the dynamic boundary condition Eq. (8). ${ }^{20}$

In the following, the length scale $d$ introduced in Eq. (6) is set equal to the gas inlet diameter depicted in Fig. 2. The velocity scale $U$ for the liquid is chosen when there is no gas flow as $Q_{\text {in }} / A_{\text {or }}$, where $A_{\text {or }}=\pi(\alpha d / 2)^{2}$ is the area of the orifice cross section. In terms of these scales, the dimensionless inlet velocity is given as

$$
\tilde{U}_{\text {in }}(s)=\frac{3 \alpha^{2}}{\left(\frac{d_{\text {in }}}{d}\right)^{2}+3 \frac{d_{\text {in }}}{d}} s(1-s),
$$

where $s \in[0 ; 1[$ is a curve parameter on the inlet boundary. The dimensionless, constant pressure $\widetilde{p}_{2}$ in the gas and the capillary number $\mathrm{Ca}$ are defined as
TABLE I. Typical values of the parameters used in the experiments on the planar flow-focusing device. ${ }^{12,13}$

\begin{tabular}{lc}
\hline \hline Parameter & Typical value \\
\hline$d$ & $200 \mu \mathrm{m}$ \\
$Q_{\text {in }}$ & $0.05 \mu \mathrm{L} / \mathrm{s}$ \\
$p_{\text {gas }}$ & $5-100 \mathrm{kPa}$ \\
$\mu$ & $1-10 \mathrm{mPa} \mathrm{s}$ \\
$\gamma_{\mathrm{lg}}$ & 30 and $73 \mathrm{~mJ} / \mathrm{m}^{2}$ \\
$U$ & $2 \mathrm{~mm} / \mathrm{s}$ \\
$\operatorname{Re}$ & $0.02-0.2$ \\
$\mathrm{Ca}$ & $10^{-3}-10^{-1}$ \\
\hline \hline
\end{tabular}

$$
\begin{aligned}
& \mathrm{Ca} \equiv \frac{\mu U}{\gamma_{\mathrm{gg}}}, \\
& \widetilde{p}_{2} \equiv \frac{p_{\mathrm{gas}} d}{\mu U} .
\end{aligned}
$$

Typical parameter values for the various physical quantities are listed in Table I. In terms of the rescaled variables and parameters, the dynamic boundary condition Eq. (8) in component form is

$$
\begin{aligned}
& \Gamma_{11} n_{1}+\Gamma_{12} n_{2}=\widetilde{r}\left(\frac{1}{\mathrm{Ca}} \widetilde{\kappa}-\widetilde{p}_{2}\right) n_{1}, \\
& \Gamma_{21} n_{1}+\Gamma_{22} n_{2}=\widetilde{r}\left(\frac{1}{\mathrm{Ca}} \widetilde{\kappa}-\widetilde{p}_{2}\right) n_{2},
\end{aligned}
$$

where $\widetilde{\kappa}$ is the dimensionless curvature and $\Gamma_{i j}$ are the components of the modified Cauchy stress matrix in Eq. (7).

The liquid-gas interface is represented as a parametric curve $\widetilde{\boldsymbol{x}}(S)=[\widetilde{z}(S), \widetilde{r}(S)]$, where $S$ is the arc-length parameter scaled by the total length of the given interface. The dimensionless curvature $\widetilde{\kappa}$ of the interface is given by the nonlinear expression

$$
\widetilde{r} \widetilde{\kappa}=\tilde{r} \frac{\ddot{z} \ddot{r}-\ddot{r} \ddot{z}}{\left(\dot{r}^{2}+\dot{z}^{2}\right)^{3 / 2}}-\frac{\dot{z}}{\left(\dot{r}^{2}+\dot{z}^{2}\right)^{1 / 2}},
$$

where a dot is the derivative with respect to $S$, and we have multiplied with $\widetilde{r}$. The last term in the expression is equal to the $r$ component $n_{2}$ of the surface normal and is related to the curvature in the azimuthal direction.

\section{Numerical method}

As mentioned above the Stokes problem effectively reduces to a quasistationary problem, where at any given time the flow field $(u, v, p)$ is a function of the shape of the liquidgas interface. This fact is utilized for the numerical treatment of the problem. The problem is solved numerically by using the commercial finite-element program FEMLAB together with a MATLAB code that we have developed comprising the time evolution and numerical schemes for representing the curvature of the interface. The method is inspired by the boundary-integral method ${ }^{2,3}$ and classical finite-element methods. ${ }^{21,22}$ At every time step, we begin with a given po- 


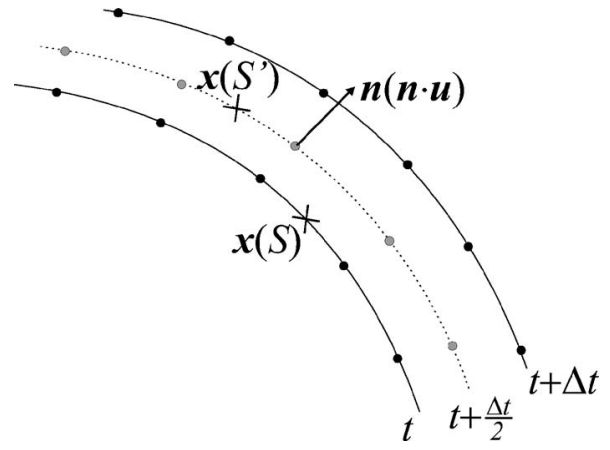

FIG. 3. Schematic representation of an interface segment with associated grid points. The interface is represented by a curve $\boldsymbol{x}(S)$ at time $t$ and $\boldsymbol{x}\left(S^{\prime}\right)=\boldsymbol{x}\left[f^{-1}(S)\right]$ at the intermediate time $t+\Delta t / 2$. The gradient $\boldsymbol{n}(\boldsymbol{n} \cdot \boldsymbol{u})$ of the interface position $x(S)$ used to evolve the interface to time $t+\Delta t$ is derived at the intermediate time, according to a Runge-Kutta scheme.

sition of the liquid-gas interface and solve for the flow field, and based on the new velocity information at the interface, the position of the liquid-gas interface is updated.

To evolve the interface in time we use a second-order Runge-Kutta time step algorithm. This algorithm is extended such that the elongation of the free interface is taken into account. The interface is given by the curve $\boldsymbol{x}(S)$, which is described by a cubic spline that interpolates all the mesh points on the interface. Points on the boundary are only moved normal to the interface such that Eq. (9) is transformed to

$$
\frac{d \boldsymbol{x}(S)}{d t}=\left.(\boldsymbol{u} \cdot \boldsymbol{n}) \boldsymbol{n}\right|_{\boldsymbol{x} \in \partial \Omega_{i}},
$$

where $\boldsymbol{n}$ is the surface normal. Whenever the interface is evolved the finite-element mesh is regenerated to find a new solution of the fields, and as a consequence the boundary points shift from one time step to the next. Hence, to determine the correct Runge-Kutta gradient $(\boldsymbol{u} \cdot \boldsymbol{n}) \boldsymbol{n}$, we have introduced a mapping $S=f\left(S^{\prime}\right)$, which ensures that the correct gradients, found at time $t+\Delta t / 2$, are used at the original interface points at time $t$ to evolve the interface to the next time $t+\Delta t$; see the sketch in Fig. 3. Moreover, the mapping also handles the problem of the interface changing length. ${ }^{23}$

The form of Eq. (7) and the dynamic boundary conditions Eqs. (13a) and (13b) are suited for direct use in FEMLAB. At each new time step the system is remeshed, a new curve parameter $S$ is found, and the flow equations with the correct boundary conditions are solved in FEMLAB. The number of points used to track the interface varies from 30, initially, up to 400 at snap-off. The number increases when narrow liquid regions appear that need to be well resolved. The curvature $\kappa$ of the interface is found by using an approximating least square cubic spline. This ensures the curvature to be smooth and it eliminates numerical noise introduced by the FEMLAB meshing algorithm. Moreover, the time step $\Delta t$ is determined such that no boundary point is moved more than one-third of the local mesh size.

A series of tests was performed to verify the time evolution algorithm and the code in general. The time evolution algorithm was compared with a simple Euler method, where

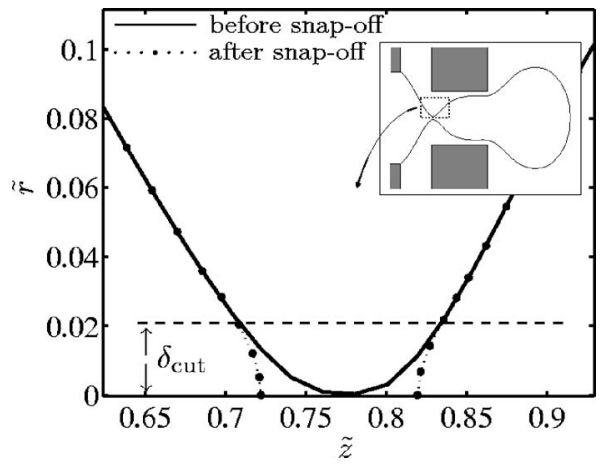

FIG. 4. The shape $\widetilde{r}(\widetilde{z})$ of the bubble just before snap-off (solid line) and the new shape after snap-off (dotted line and black points). The length scale $\delta_{\text {cut }}$ (dashed line) determines where to cut the initial shape.

the time step was chosen to ensure stability. ${ }^{20,23,24}$ The solutions were identical, but our method was ten times faster. The code was also tested for stability regarding mesh size and the choice of basis functions. Finally, the code was tested on three specific problems and performed very well in all three cases: (1) a drop in an extensional flow, ${ }^{25-27}$ (2) the coalescence of two cylindrically shaped liquid bodies, ${ }^{28}$ and (3) the steady-state shape of a gas bubble translating in a liquidfilled capillary. ${ }^{29}$ Ample details about all the tests are found in Ref. 30.

\section{Snap-off mechanism}

When a bubble snap-off occurs, the curve representing the shape of the gas thread (solid line in Fig. 4) has to be divided into two curves (dotted line in Fig. 4). In this model we define a length scale $\delta_{\text {cut }}$ (marked by the dashed line in Fig. 4) that determines where to cut the original shape. The curve is cut at the nearest interface grid point and a cubic spline with vertical tangent at $r=0$ caps the gas-jet and the new bubble. The spatial resolution of the dynamics near the breakup are restricted by the mesh size $h_{m}$; hence, we have chosen $\delta_{\text {cut }}>2 h_{m}$. This method might seem crude; however, the specific shape near the breaking point does not influence the overall dynamics of the system. Just after the snap-off discontinuities are registered in the liquid pressure at the orifice region as the breakup event is a singular event in itself.

\section{RESULTS AND DISCUSSION}

The time-dependent dynamics of the liquid-gas system evolving in the flow-focusing device sketched in Fig. 1 are controlled by many parameters. In the following, we have restricted our analysis to the dependence on four parameters: (1) the pressure of the gas thread; (2) the inlet liquid flowrate $Q_{\text {in }}=A_{\text {or }} U$ (3) one geometry component, namely, the outlet channel radius $H$ (see Fig. 2); and (4) the liquid-gas interfacial tension $\gamma_{\mathrm{lg}}$ characterized by the capillary number $\mathrm{Ca}$. The rest of the geometry parameters depicted in Fig. 2 are constant and set to $\left(\alpha, \beta, \gamma, d_{\text {in }} / d, W / d\right)$ $=(0.5,1.5,2,1,15)$. An important parameter to control and observe experimentally is the volume $V_{b}$ of the created bubbles. The bubble volume is also the focus of our analysis as it is straightforward to determine numerically. 


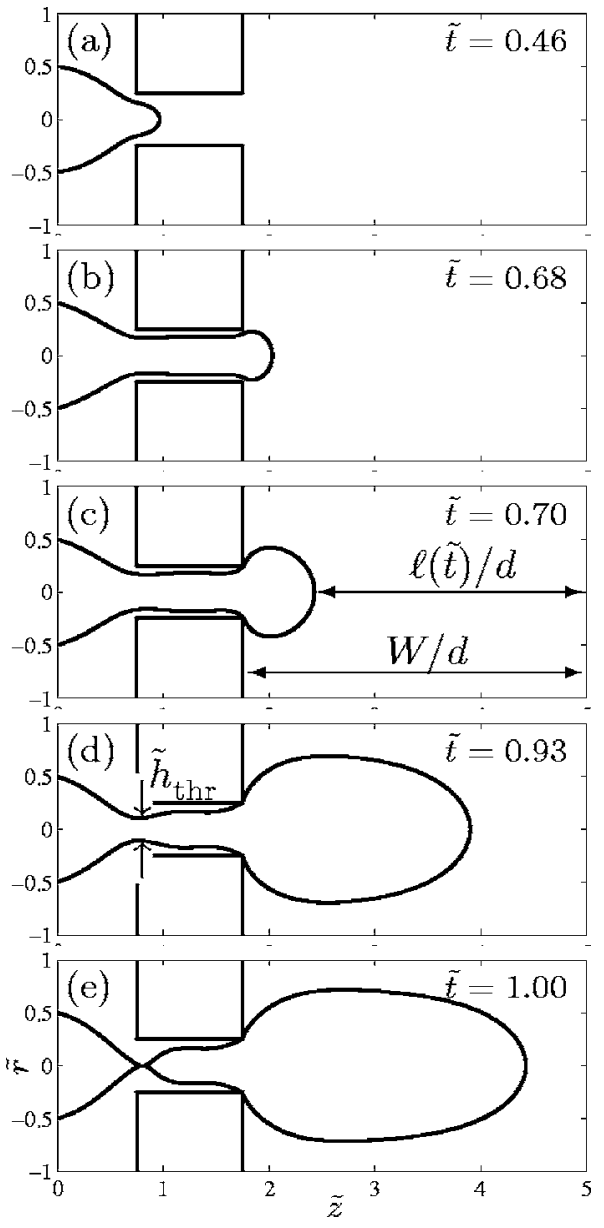

FIG. 5. A sequence of five snapshots from the simulations representing the creation of a bubble: (a) the initial insertion of the gas thread into the orifice, (b) the exit of the gas thread from the orifice region, (c) the blocking of the inlet liquid flow to the outlet, (d) the controlled collapse of the gas thread, and finally (e) the snap-off event where $\tilde{h}_{\text {thr }}=0$. The length $\ell(\widetilde{t})$ is measured from the tip of the bursting head of the gas thread to the outlet where $\tilde{p}$ $=0, W$ is the length of the outlet region, and $\tilde{h}_{\mathrm{thr}}=h_{\mathrm{thr}} / d$ is the minimal dimensionless diameter of the gas thread. The simulations are for $\left(\tilde{p}_{2}, \mathrm{Ca}, H / d\right)=(350,0.1,1)$.

We begin by studying various aspects of the creation of the first bubble in a bubbling sequence and show how $V_{b}$ scales with the four parameters analyzed. Finally, we study a sequence of four bubbles in a specific geometry. Our results on the axisymmetric geometry are compared to experimental findings on the planar geometry given in Refs. 12 and 13.

\section{A. Bubble growth morphology}

The snapshots depicted in Figs. 5(a)-5(e) represent the creation of a typical bubble. Panel (a) shows the initial insertion of the gas thread into the orifice, (b) the exit from the orifice region of the bursting head of the gas thread, (c) the blocking of the inlet liquid flow as the bubble inflates into the outlet channel, (d) appearance of a clear collapse region and collapse of the gas thread, and finally (e) the snap-off event where the thread radius vanishes $\left(\tilde{h}_{\mathrm{thr}}=0\right)$. In this case, the flow and geometry parameters are $\left(\tilde{p}_{2}, \mathrm{Ca}, H / d\right)$ $=(350,0.1,1)$, and the total time simulated is $\tilde{t}=1.0$. The time-dependent distance from the tip of the bursting head of

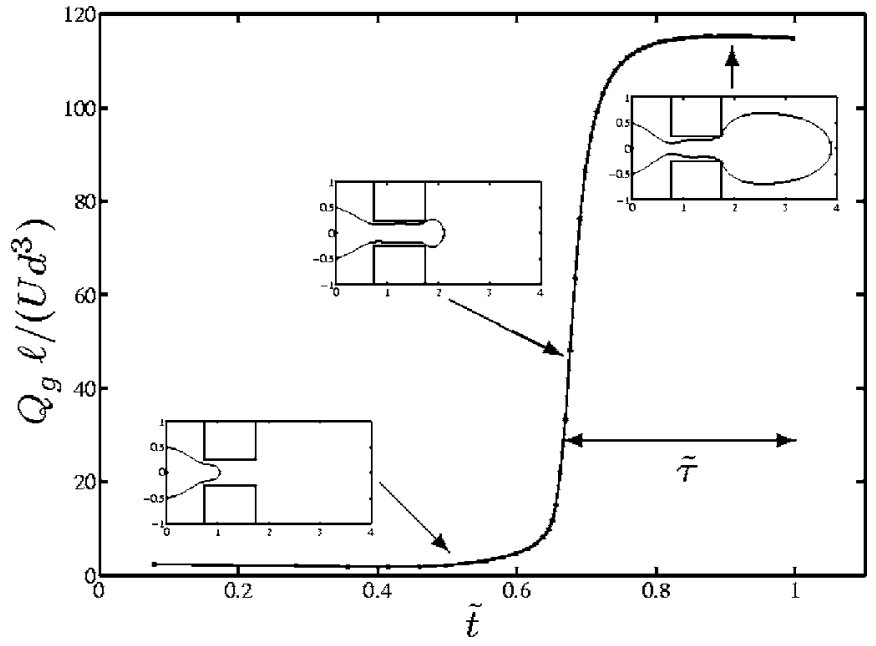

FIG. 6. Plot of $Q_{g} \ell /\left(U d^{4}\right)$ as function of time for $\mathrm{Ca}=0.1, \tilde{p}_{2}=350$, and $H / d=1$. The gas dimensionless flow-rate is found numerically and calculated as $\widetilde{Q}_{g}=d \widetilde{V}_{g} / d \widetilde{t}$, where $\widetilde{V}_{g}$ is the volume of the gas to the right of the gas inlet at $\widetilde{z}=0$.

the gas thread to the outlet, where $\tilde{p}=0$, is denoted $\ell=\ell(t)$, while $W$ is the constant length of the outlet region [see Fig. $5(\mathrm{c})]$. The length $\tilde{h}_{\mathrm{thr}}=h_{\mathrm{thr}} / d$ is the dimensionless minimal diameter of the gas thread and is located at $\tilde{z}=z_{\text {thr }} / d$. The volume $V_{b}$ of a bubble at snap-off is simply calculated as

$$
V_{b}=\pi \int_{z=z_{\mathrm{thr}}}^{z_{\max }} r^{2}(z) d z,
$$

where $z_{\max }$ is the $z$ coordinate of the front of the gas thread and $r(z)$ is a representation of the interface curve. We will be concerned mainly with bubbles of relatively large volume, for which snap-off occurs when $\ell<W$. For some choice of parameters, small bubbles may snap-off when they are still in the orifice; i.e., when $\ell>W$. At the time of snap-off, $z_{\text {thr }}$ is generally located at the left end of the orifice.

\section{B. Bubble growth time $\tau$}

The time it takes a bubble to grow is denoted $\tau$. It is defined as the time between the onset of the gas thread collapse [Fig. 5(c)], when the emerging bubble blocks the liquid flow, and the snap-off of the bubble [Fig. 5(e)]. The collapse is characterized by a rapid decrease of $h_{\mathrm{thr}}$ and a rapid increase of $Q_{g}$, as illustrated in Fig. 6. When the blocking of the orifice sets in, the liquid flow fills the orifice volume $V_{\text {or }}=A_{\text {or }} \gamma d$ at the rate $Q_{\text {in }}$, squeezes the gas thread, and induces the collapse leading to bubble snap-off. As described in Ref. 13, for a fixed orifice geometry and surface tension, the bubble growth time $\tau$ therefore scales as

$$
\tau \propto \frac{V_{\text {or }}}{Q_{\text {in }}} \propto \frac{1}{U} .
$$

Numerically, we found that for varying values of $\mathrm{Ca}$ and $\widetilde{p}_{2}$, the dimensionless time $\tilde{\tau}$ was constant $(\sim 0.3)$, and hence that $\tau \propto d / U$. For a constant geometry, the numerics confirmed the predicted linear dependence. 


\section{Gas flow-rate $Q_{g}$}

The gas flow-rate $Q_{g}$ is not set explicitly, but it can be determined as follows. When the emerging gas bubble begins to block the orifice, it interrupts the liquid flow from the inlet to the outlet. The liquid flow-rate $Q_{\text {out }}$ at the outlet is therefore, by volume conservation, equal to the flow-rate $Q_{g}$ of the gas. Assuming the liquid flow in the outlet region to be a Poiseuille flow, we obtain

$$
Q_{g}=Q_{\text {out }}=\frac{\pi}{8} \frac{1}{\mu} G H^{4} \propto G H^{4},
$$

where $G$ is the pressure gradient. A good approximation for $G$ is given by the pressure drop from the tip of the thread to the outlet divided by the distance $\ell$ [see Fig. 5(c)]. As the emerging bubble blocks the liquid flow, the pressure in front of it is approximately $p_{\mathrm{gas}}-\gamma_{\mathrm{lg}} \kappa$, where $\kappa \equiv 2 / R$. If we furthermore assume that the bubbles being created are big, i.e., of size comparable to $H$, we have

$$
G \approx \frac{1}{\ell}\left(p_{\text {gas }}-\frac{2 \gamma_{\mathrm{lg}}}{H}\right)
$$

where the radius of curvature $R \approx H$ because of the axisymmetric nature of the geometry studied. Combining Eqs. (18) and (19) leads to

$$
Q_{g} \ell \propto H^{4}\left(p_{\text {gas }}-\frac{2 \gamma_{\mathrm{lg}}}{H}\right) .
$$

Consequently, for constant geometry, gas pressure, and surface tension, we expect $Q_{g} \ell$ to be constant. To verify this expression numerically, the length $\ell$ is found as the difference between $W$ and the position of the gas front, and $Q_{g}$ is found as $d V_{g} / d t$, where $V_{g}$ is the volume of the gas. The dimensionless quantity $Q_{g} \ell /\left(U d^{3}\right)$ is plotted as function of dimensionless time $\tilde{t}$ in Fig. 6, where we see that $Q_{g} \ell$ indeed is constant for $\tilde{t}>0.7$ after the gas bubble begins to block the orifice. From Fig. 6, it is also seen how $\tilde{\tau}$ was determined numerically as $Q_{g} \ell(\widetilde{t})$ changes abruptly when the blocking of the orifice is initiated.

\section{Gas-thread collapse speed $u_{s}$}

The collapse speed of the gas thread just before snap-off is defined as

$$
\left.u_{s} \equiv \frac{d h_{\mathrm{thr}}}{d t}\right|_{t=t_{0}}
$$

where $t_{0}$ is the time of snap-off. Figure 7 shows $u_{s} / U$ as function of $1 / \mathrm{Ca}$. The inset shows the dimensionless thread thickness as function of time $\widetilde{h}_{\text {thr }}(\widetilde{t})$, which corresponds to the evolution from Fig. 5(d) and 5(e). The collapse speed $u_{s} / U$ is determined by a linear fit to the curve $\widetilde{h}_{\mathrm{thr}}(\widetilde{t})$ near its intersection with the $\widetilde{t}$ axis. The spread in the calculated values is due to uncertainties in this fit. It is interesting to find that $u_{s} / U$ depends linearly on $1 / \mathrm{Ca}$, for $\mathrm{Ca}>0.1$. This means that $u_{s} \propto \gamma_{\mathrm{lg}} / \mu$. Studies of breakup of viscous threads in axisymmetric geometries (see Refs. 8-10 and 32) show that just

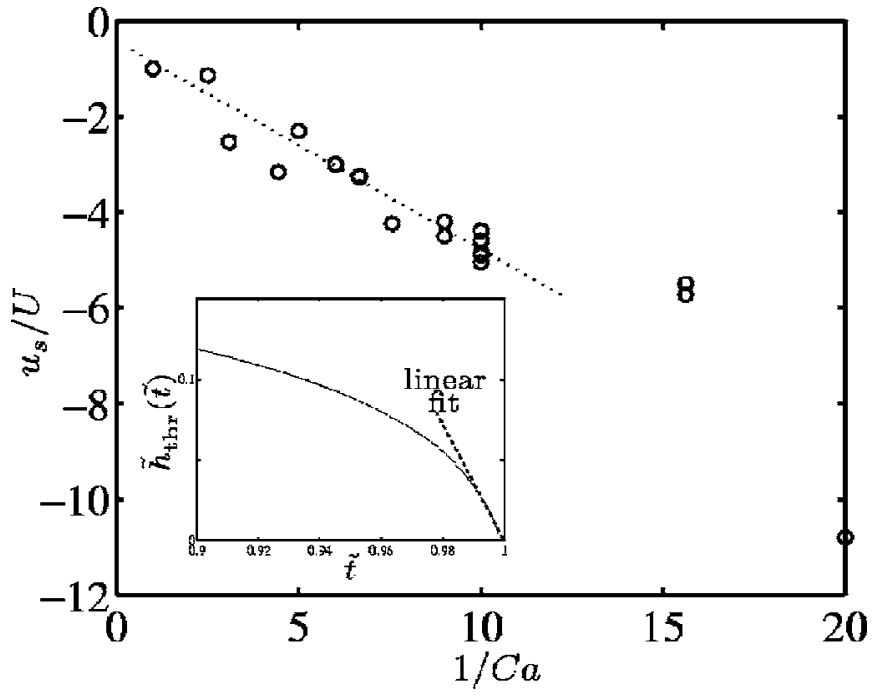

FIG. 7. Collapse speed $u_{s} / U$ at snap-off of the gas thread as function of Ca. The inset depicts $\tilde{h}_{\text {thr }}(\widetilde{t})$ corresponding to the evolution from Figs. 5(d) and 5(e). The dashed line corresponds to the linear fit made to determine $u_{s} / U$.

before snap-off the thread thickness $h_{\mathrm{thr}} \propto\left(t_{0}-t\right) \gamma_{\mathrm{lg}} / \mu$, which agrees with our numerical findings.

The numerically determined time-dependent evolution of $h_{\text {thr }}$ is qualitatively similar to the one observed experimentally in Ref. 13. The gas thread thickness is initially nearly constant before it rapidly snaps during the time $\tau$. However, the detailed evolution of the shape of the interface is different in the two cases: In the experimental work, ${ }^{13}$ the collapse region of the gas thread was seen to extend from the gas inlet to the right of the orifice. In our axisymmetric model, the collapse region is localized to an area just left of the orifice [see Figs. 5(d) and 5(e)]. We speculate that this disagreement is due to the anisotropic confinement of the gas thread in the planar geometry of the experiments versus the axisymmetric confinement in our model. This most probably influences the dynamics of the collapse. ${ }^{31}$

\section{E. Bubble volume $V_{b}$}

The volume of a bubble at snap-off is given by $V_{b}$ in Eq. (16). It is proportional to the time $\tau$ that the gas thread stays open times the flow-rate $Q_{g}$ of the gas. Combining Eqs. (17)-(19) and having $\ell$ nearly constant leads to

$$
V_{b} \propto \frac{H^{4}}{\mu Q_{\text {in }}}\left(p_{\text {gas }}-\frac{2 \gamma_{\mathrm{lg}}}{H}\right) .
$$

For the experimental results, presented in Refs. 12 and 13, it was determined that for a fixed geometry and surface tension the volume of the bubbles is proportional to the ratio $p_{\text {gas }} / \mu Q_{\text {in }}$. This scaling is directly given by Eq. (22) for constant geometry. We also confirm this scaling with our numerical results shown in Fig. 8, where, for $H / d=0.8,1$, and 1.2 (fixed geometry), the dimensionless volume $V_{b} / d^{3}$ of the bubble at snap-off is seen to scale as $\widetilde{p}_{2} \propto p_{\text {gas }} / \mu Q_{\text {in. }}$. Note that there is a deviation from this scaling for small bubbles that do not emerge from the orifice before snap-off, i.e., when $\ell>W$, and our assumptions do not hold. 


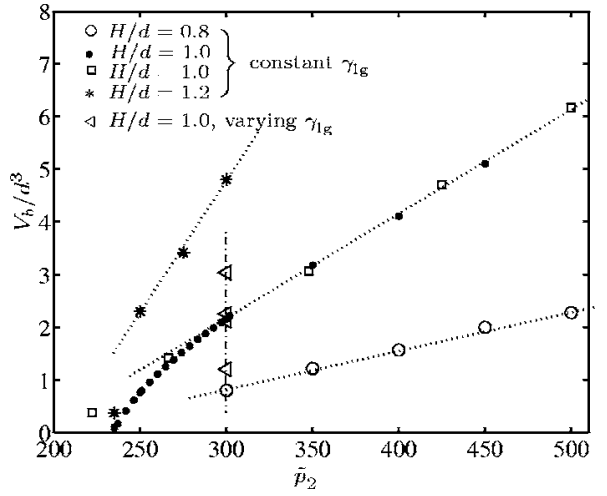

FIG. 8. The dimensionless volume of the first bubble as function of the parameter $\widetilde{p}_{2}$, for three values of the outlet channel radius $H / d=0.8,1.0$, and 1.2 for constant surface tension $\gamma_{\mathrm{lg}}$. Moreover, the triangles show a variation of the capillary number $\mathrm{Ca}$; i.e., a change in the surface tension for fixed $H / d, p_{\text {gas }}$, and $Q_{\text {in }}$. See Fig. 10 for further details.

In Fig. 9 we have depicted $V_{b} d / H^{4}$ as function of $\tilde{p}_{2}$ and see that they fall on one line; hence, that $V_{b} \propto p_{\text {gas }} H^{4} / \mu Q_{\text {in }}$. This scaling also follows directly from our general expression Eq. (22).

Finally, we study the scaling of the bubble volume as function of the capillary number $\mathrm{Ca}$ for constant dimensionless pressure $\tilde{p}_{2}$. In Fig. 10, the volume $V_{b} / d^{3}$ is plotted as function of $\mathrm{Ca}$ for $\tilde{p}_{2}=300$ and $H / d=1$, meaning that we plot it as function of $p_{\text {gas }} d / 300 \gamma_{\mathrm{lg}}$. We note a linear relation for values of $\mathrm{Ca}$ larger than some critical value $C a^{*} \approx 0.1$. In this regime, we have $V_{b} \propto \mu U / \gamma_{\mathrm{lg}}$, or after multiplication by the constant dimensionless pressure $V_{b} \propto p_{\text {gas }} d / \gamma_{\mathrm{lg}}$ for $p_{\text {gas }} d / \gamma_{\mathrm{lg}}$ $>30$. This last critical value relates to whether a bubble is large $(\ell<W)$, or is small $(\ell>W)$ when it snaps off (see Fig. $5)$. When the gas pressure cannot overcome the YoungLaplace pressure in the orifice the bubble snap-off occurs for $\ell>(W+\gamma d)$, and we have $p_{\text {gas }}<2 \gamma_{\mathrm{lg}} /(\alpha d / 2)$, yielding $p_{\text {gas }} d / \gamma_{\text {lg }}<8$. Values of $p_{\text {gas }} d / \gamma_{\text {lg }}$ between 8 and 30 correspond to the situation where the gas front is in the orifice or just left of it when bubble snap-off occurs. For values of $p_{\text {gas }} d / \gamma_{\text {lg }}<8$ corresponding to $\mathrm{Ca}<0.05$, no bubble is created $\left(V_{b}=0\right)$ and we find a steady state solution of the inter-

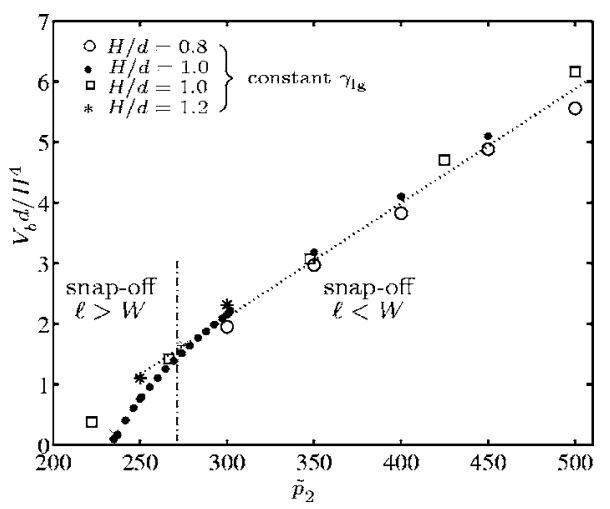

FIG. 9. Plot of the volume $V_{b} / d^{3}$ scaled with the outlet channel radius $(H / d)^{4}$ as a function of $\widetilde{p}_{2}$ for constant surface tension $\gamma_{\mathrm{lg}}$. Note that the three lines sketched in Fig. 8 now merge, as predicted by Eq. (22). The vertical dashed line indicates the transition from bubbles snap-off when $\ell$ $>W$ and $\ell<W$.

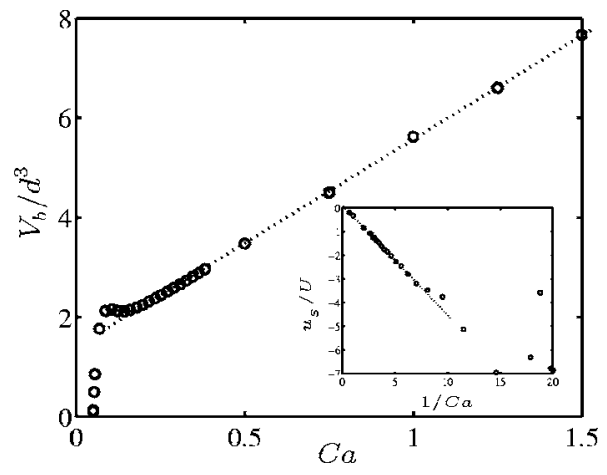

FIG. 10. The volume $V_{b}$ of the first bubble as function of the capillary number $\mathrm{Ca}$ for constant pressure $\tilde{p}_{2}=300$ and radius $H / d=1$. The inset shows the collapse speed $u_{s} / U$ as a function of $1 / \mathrm{Ca}$.

face. In the opposite limit of very large values of the capillary number, a continuous gas jet is streaming from the gas outlet as observed experimentally by, e.g., Sevilla et al. ${ }^{33}$

The inset in Fig. 10 represents the velocity $u_{s} / U$ as function of $1 / \mathrm{Ca}$ for the same parameters. From the figure it is seen that $u_{s} / U \propto 1 / \mathrm{Ca}$. For $\mathrm{Ca}<\mathrm{Ca}^{*}$, the linearity in $1 / \mathrm{Ca}$ is not clear; this scattering of the data points could be due to numerical noise.

\section{F. Multi-bubble sequence}

In this section we describe a system where four bubbles are created in a sequence. There are three snap-off events and the simulation ends just before the fourth bubble snaps off. The parameters for this model are: $\mathrm{Ca}=0.1, \widetilde{p}_{2}=320, W / d$ $=15, H / d=0.6,(\alpha, \beta, \gamma)=(0.5,1.5,2)$. The total computation time for the simulation was one week on an Intel Pentium $4 ®, 3.2 \mathrm{GHz}$ with $1 \mathrm{~GB}$ of RAM.

Snapshots of the bubble shape at selected times $\tilde{t}$ are depicted in Fig. 11. The size of the bubbles are measured in terms of $\tilde{p} V / d^{3}$, which is constant for each of the four bubbles and has the value 80.8, 232.7, 208.1, and 195.3, respectively. The data indicate that the system exhibits a transient behavior before reaching a stable period-one bubbling. The snapshots at Figs. 11(b), 11(d), 11(f), and 11(h) are taken just before a bubble snap-off occurs. It is clearly seen how a preceding bubble shrinks when a new bubble is about to be released. As discussed earlier, a bubble blocks the liquid flow out of the orifice before it snap-off. The blocking occurs on a short time scale leading to an immediate pressure increase in the liquid in front. The pressure in the liquid in front of the bubble equals the pressure $\tilde{p}_{2}$ in the bubble minus the curvature contribution [see Eq. (19)]. The preceding bubbles cannot escape because of the large viscous drag in the Stokes-flow regime. This results in a very pronounced compression of the preceding bubbles. The compression is seen graphically in Fig. 11: the first bubble is, e.g., seen to shrink from Figs. 11(c) and 11(d) and then enlarge from 11(d) and 11(e).

The volumes $V_{b} / d^{3}$ of the three released bubbles are plotted as function of time in Fig. 12(a). From the graph it is seen how preceding bubbles are compressed when a new bubble is released. The bubbles are released at the times 
(a)

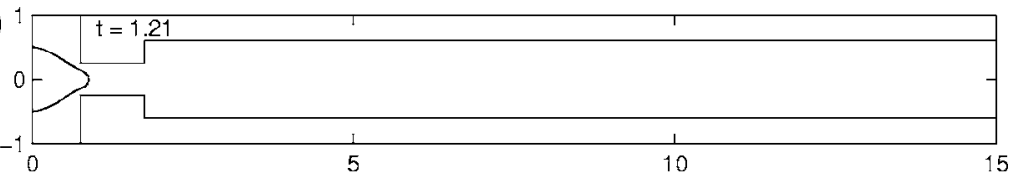

(b)

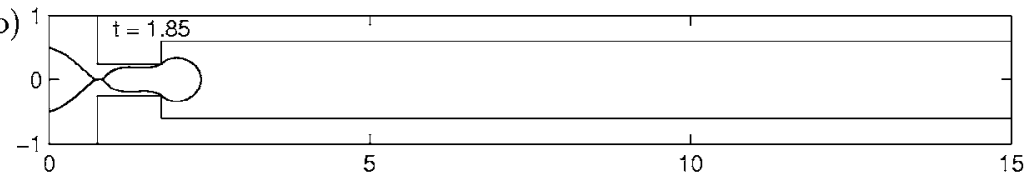

(c)

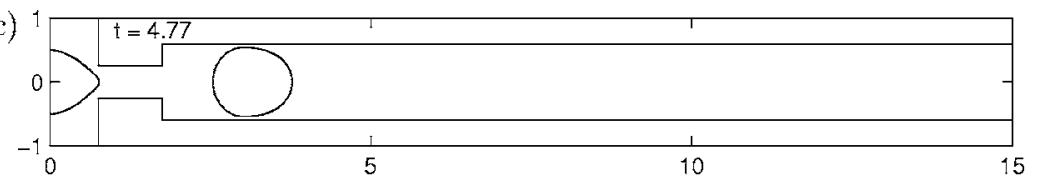

(d)

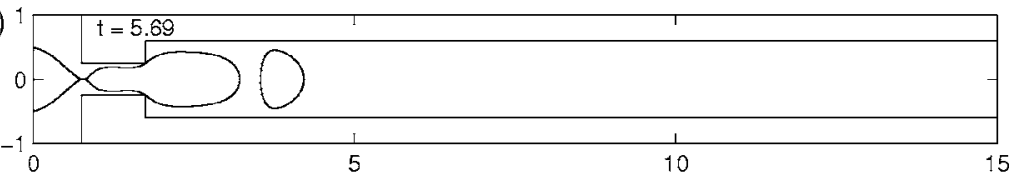

(e)

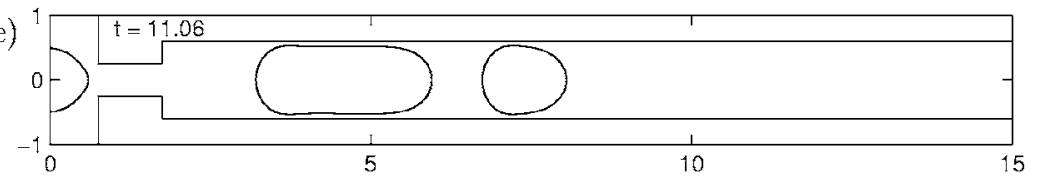

(f)

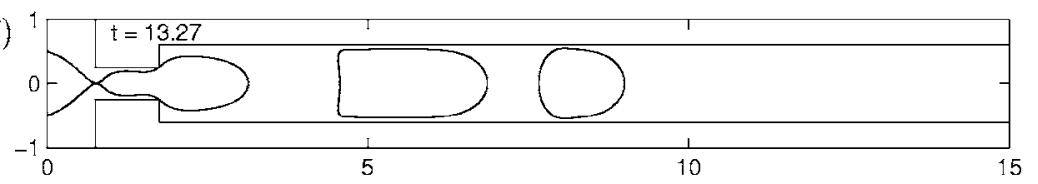

(g)
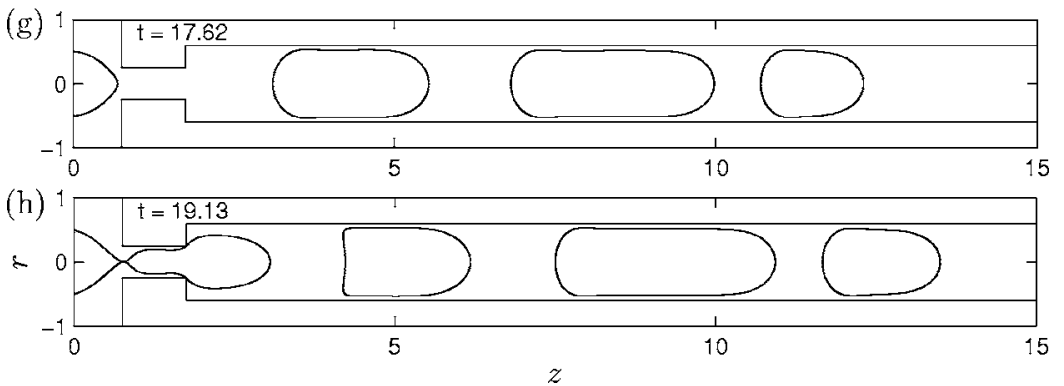

FIG. 11. A sequence showing the generation of the first four bubbles. The bubble shapes are depicted at different selected times $\tilde{t}$. Snapshots (b), (d), (f), and (h) are taken just before a bubble snap-off event. marked by the vertical dotted lines. These correspond to the events shown in Figs. 11(b), 11(d), and 11(f). In Fig. 12(b), the mean pressure on a bubble

$$
\left\langle\widetilde{p}_{i}\right\rangle=\int_{\partial \Omega_{i}} \boldsymbol{n} \cdot \boldsymbol{\sigma} \cdot \boldsymbol{n} d A\left(\int_{\partial \Omega_{i}} d A\right)^{-1}
$$

is depicted as function of time. We clearly see how the volume of a bubble increases as the pressure around it decreases and vice versa. In our model the compressibility of the bubbles is further enhanced as we have a relatively short outlet channel and because the reference pressure at the outlet is zero. ${ }^{34}$ In experiments where the outlet channel is very long compared to a typical bubble length the compressibility effects are not very pronounced. The bubbles are, however, seen to expand as they travel far in the outlet. ${ }^{35}$

In Fig. 13 the gas-thread thickness $\widetilde{h}_{\text {thr }}$ is plotted as function of the time $\tilde{t}-\widetilde{t}_{0}$. We see that for all four bubbles $\widetilde{h}_{\mathrm{thr}}(\widetilde{t})$ has the same shape and hence the same collapse speed $d h_{\mathrm{thr}} / d t$. This indicates that the snap-off event is local and not influenced by the hydrodynamic interactions of bubbles further downstream. This results is supported by experimen- tal results, ${ }^{13}$ in terms of the so-called flow-rate controlled breakup of the gas thread: the collapse velocity of the liquid thread is only a function of $Q_{\text {in }}$ for a given orifice geometry.

\section{CONCLUSION}

In this work we have performed a numerical study of the dynamics of bubble formation in an axisymmetric flowfocusing device in the Stokes-flow limit. For this purpose a generic numerical model for solving free-surface flows in an axisymmetric geometry was developed. The model is based on a second-order Runge-Kutta time integration algorithm and free-surface scheme implemented in MATLAB, which are coupled to the commercial finite-element solver FEMLAB.

The analysis of the flow-focusing device was restricted to four parameters: the inlet liquid flow-rate $Q_{\text {in }}$, the gas pressure $p_{\text {gas }}$, the outlet channel radius $H$, and the liquid-gas surface tension $\gamma_{\mathrm{lg}}$. Based on these parameters, we derive scaling laws for the volume $V_{b}$ of a created bubble and for the gas flow-rate $Q_{g}$. By physical analysis and numerical simulation, we have shown that the bubble growth time $\tau$ scales proportionally to the inverse liquid flow rate $1 / Q_{\text {in }}$. 
(a)

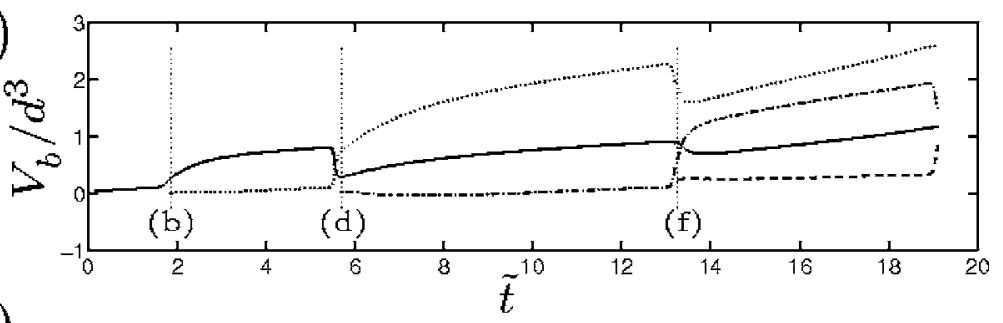

(b)

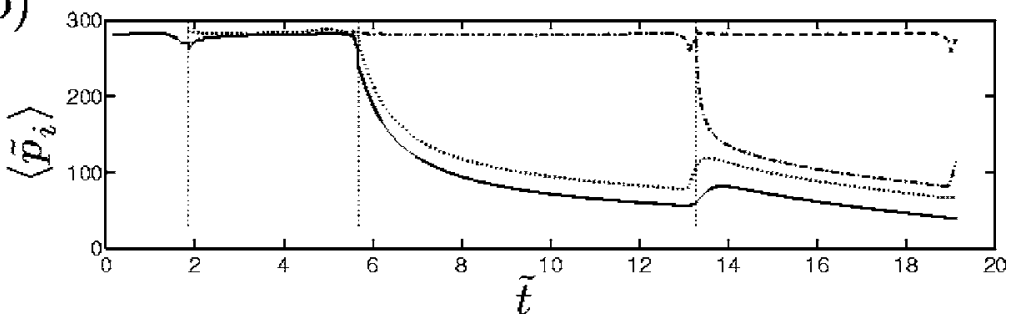

FIG. 12. (a) The volume $V_{b} / d^{3}$ of the four bubbles as function of time $\widetilde{t}$ and (b) the mean pressure $\left\langle\widetilde{p}_{i}\right\rangle$ on the bubble surface as function of time. In all the bubbles, $\widetilde{p} V / d^{3}$ is constant. The vertical dotted lines represent the snapshots (b), (d), and (f) from Fig. 11.
The collapse speed $u_{s}$ of the gas thread immediately prior to the snap-off event was found to be proportional to the ratio $\gamma_{\mathrm{lg}} / \mu$. This scaling was not observed experimentally, ${ }^{92,13}$ but is supported by other studies of the breakup of viscous axisymmetric threads. The detailed behavior of the thread thickness as function of time $h_{\mathrm{thr}}(t)$ deviates from the experimental data. We speculate that this difference in the collapse dynamics is due to the difference in geometry of the devices. In the experiments the gas thread is confined anisotropically, while it is confined equally from all sides in our axisymmetric model. In the experimental work ${ }^{13}$ the collapse region of the gas thread was seen to extend from the gas inlet to the right of the orifice. In our axisymmetric model the collapse region is localized to an area just left of the orifice [see Figs. 5(d) and 5(e)]. In the simulated geometry the thread can minimize surface area (energy) in two dimensions simultaneously as it is not restricted by a top and bottom lid as in the planar case. ${ }^{31}$

The above-mentioned scaling law for the bubble volume is presented in Eq. (22). For a constant geometry, it reduces to the scaling law $V_{b} \propto p_{\text {gas }} / \mu Q_{\text {in }}$ described by Garstecki et $a l .{ }^{12,13}$ We confirm this law by means of simulations for various constant values of $H$. For a varying outlet channel radius, our extended scaling law also predicts an $H^{4}$ dependence; namely, $V_{b} \propto H^{4} p_{\text {gas }} / \mu Q_{\text {in }}$. We confirm this dependence by

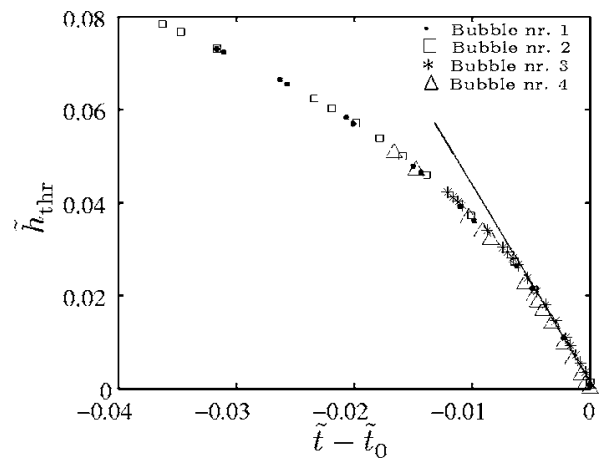

FIG. 13. The gas-thread thickness $\widetilde{h}_{\text {thr }}$ as a function of the time $\tilde{t}-\widetilde{t}_{0}$. The evolution for the four bubbles are identical. The full line represents the region where the gradient $d h_{\mathrm{thr}} / d t$ is determined, just before snap-off. means of the numerical results presented in Fig. 9. Our numerical investigation further reveals that critical values exist for the dimensionless parameters $\tilde{p}_{2}$ and $\mathrm{Ca}$ determining where the bubble is located when snap-off occurs. These values are determined by the ratio between the surfacetension-dependent Young-Laplace pressure and the gas pressure.

Using a simple mechanism to account for the snap-off we model a sequence of four consecutive bubbles and show that the flow-focusing device does not produce bubbles of constant volume straight away. The results suggest that there is a certain transient behavior before reaching steady state. Bubbles that are located upstream of the orifice influence the pressure drop in the outlet channel. The pressure gradient along the outlet channel is an important factor in the scaling of the bubble volume. Bubbles of constant volume may hence only be created when a constant pressure drop exists in the outlet. This could, for example, be the case if the outlet region is large compared to the bubbles or if large bubbles are filling the entire outlet region, just as happens in experiments. ${ }^{12}$

In the multibubble sequence we confirm that the collapse speed of the gas thread is not influenced by hydrodynamic interactions of bubbles downstream of the snap-off event. Finally, we see a large compression of the created free gas bubbles in the Stokes-flow regime. The effect is amplified due to the numerical setup but it is also seen experimentally; however, it is much less pronounced.

The numerical model we have implemented is versatile, and consequently the work presented in this paper facilitates the study of other features in flow-focusing devices. This could include other geometries of the orifice, liquid-liquid systems, or even non-Newtonian fluids. Results from such studies would be very useful when designing future experiments.

\section{ACKNOWLEDGMENTS}

We would like to thank Piotr Garstecki for many inspiring discussions and for sharing his experimental results with us at an early stage. H. A. S. thanks the Harvard MRSEC 
COMR-02/3805 and F. Jousse and colleagues at Unilever for support.

${ }^{1}$ T. W. Shield, D. B. Bogy, and F. E. Talke, "Drop formation by DOD ink-jet nozzles: A comparison of experiment and numerical simulation," IBM J. Res. Dev. 31, 96 (1987).

${ }^{2}$ C. Pozrikidis, Boundary Integral and Singularity Methods for Linearized Viscous Flows (Cambridge University Press, Cambridge, England, 1992).

${ }^{3}$ D. F. Zhang and H. A. Stone, "Drop formation in viscous flows at a vertical capillary tube," Phys. Fluids 9, 2234 (1997).

${ }^{4}$ M. Muradoglu and H. A. Stone, "Mixing in a drop moving through a serpentine channel: A computational study," Phys. Fluids 17, 73305 (2005).

${ }^{5}$ T. M. Tsai and M. J. Miksis, "Dynamics of a drop in a constricted capillary tube," J. Fluid Mech. 274, 197 (1994).

${ }^{6}$ B. Ambravaneswaran, S. D. Phillips, and O. A. Basaran, "Theoretical analysis of a dripping faucet," Phys. Rev. Lett. 85, 5332 (2000).

${ }^{7}$ A. U. Chen, P. K. Notz, and O. A. Basaran, "Computational and experimental analysis of pinch-off and scaling," Phys. Rev. Lett. 88, 174501 (2002).

${ }^{8}$ J. Eggers, "Nonlinear dynamics and breakup of free-surface flows," Rev. Mod. Phys. 69, 865 (1997).

${ }^{9}$ I. Cohen, M. P. Brenner, J. Eggers, and S. R. Nagel, "Two fluid drop snap-off problem: Experiments and theory," Phys. Rev. Lett. 83, 1147 (1999).

${ }^{10}$ M. P. Brenner, J. Eggers, K. Joseph, S. Nagel, and X. D. Shi, "Breakdown of scaling in droplet fission at high Reynolds number," Phys. Fluids 9, 1573 (1997).

${ }^{11}$ S. L. Anna, N. Bontoux, and H. A. Stone, "Formation of dispersions using 'flow focusing' in microchannels," Appl. Phys. Lett. 82, 364 (2003).

${ }^{12}$ P. Garstecki, I. Gitlin, W. DiLuzio, E. Kumacheva, H. A. Stone, and G. M. Whitesides, "Formation of monodisperse bubbles in a microfluidic flowfocusing device," Appl. Phys. Lett. 85, 2649 (2004).

${ }^{13}$ P. Garstecki, H. A. Stone, and G. M. Whitesides, "Mechanism for flowrate controlled breakup in confined geometries: A route to monodisperse emulsions," Phys. Rev. Lett. 94, 164501 (2005).

${ }^{14}$ P. Garstecki, M. J. Fuerstman, and G. M. Whitesides, "Nonlinear dynamics of a flow-focusing bubble generator: An inverted dripping faucet," Phys. Rev. Lett. 94, 234502 (2005).

${ }^{15}$ A. M. Gañán-Calvo and J. M. Gordillo, "Perfectly monodisperse microbubbling by capillary flow focusing," Phys. Rev. Lett. 87, 274501 (2001).

${ }^{16}$ A. M. Gañán-Calvo, "Perfectly monodisperse microbubbling by capillary flow focusing: An alternate physical description and universal scaling," Phys. Rev. E 69, 027301 (2004).

${ }^{17}$ A. S. Utada, E. Lorenceau, D. R. Link, P. D. Kaplan, H. A. Stone, and D. A. Weitz, "Monodisperse double emulsions generated from a microcapillary device," Science 308, 537 (2005).
${ }^{18}$ MATLAB version 7 (R14), The MathWorks, Inc., Natick, MA 01760-2098; www.mathworks.com

${ }^{19}$ FEMLAB version 3.1, Comsol, Inc., Burlington, MA 01803; www.comsol.com

${ }^{20}$ T. Y. Hou, J. S. Lowengrub, and M. J. Shelley, "Removing the stiffness from interfacial flows with surface tension," J. Comput. Phys. 114, 312 (1994).

${ }^{21}$ J. N. Reddy and D. K. Gartlink, The Finite Element Method in Heat Transfer and Fluid Dynamics, 2nd ed. (CRC Press, Boca Raton, FL, 2000).

${ }^{22}$ R. D. Cook, D. S. Malkus, M. E. Plesha, and R. J. Will, Concepts and Applications of Finite Element Analysis, 4th ed. (John Wiley and Sons, New York, 2002).

${ }^{23}$ J. R. Cash and S. Semnani, "Modified Adams method for nonstiff and mildly stiff initial value problems," ACM Trans. Math. Softw. 19, 63 (1993).

${ }^{24} \mathrm{P}$. Bach and O. Hassager, "An algorithm for the use of the Lagrangian specification in Newtonian fluid mechanics and applications to freesurface flow," J. Fluid Mech. 152, 173 (1985).

${ }^{25} \mathrm{H}$. A. Stone, "Dynamics of drop deformation and breakup in viscous fluids," Annu. Rev. Fluid Mech. 26, 65 (1994).

${ }^{26}$ Y. Navot, "Critical behavior of drop breakup in axisymmetric viscous flow," Phys. Fluids 11, 990 (1999).

${ }^{27}$ B. J. Bentley and L. G. Leal, "An experimental investigation of drop deformation and breakup in steady, two-dimensional linear flows," J. Fluid Mech. 167, 241 (1985).

${ }^{28}$ R. W. Hopper, "Plane Stokes flow driven by capillarity on a free surface," J. Fluid Mech. 213, 349 (1990).

${ }^{29}$ F. P. Bretherton, "Motion of long bubbles in tubes," J. Fluid Mech. 10, 166 (1961).

${ }^{30}$ M. J. Jensen, "Numerical simulations of interface dynamics in microfluidics," Ph.D. thesis, MIC - Department of Micro and Nanotechnology, Technical University of Denmark, 2005; also available online at www.mic.dtu.dk/MIFTS

${ }^{31}$ Y. Son, N. S. Martys, J. G. Hagedorn, and K. B. Migler, "Suppression of capillary instability of a polymeric thread via parallel plate confinement," Macromolecules 36, 5825 (2003).

${ }^{32}$ J. R. Lister and H. A. Stone, "Capillary breakup of a viscous thread surrounded by another viscous fluid," Phys. Fluids 10, 2758 (1998).

${ }^{33}$ A. Sevilla, J. M. Gordillo, and C. Martínez-Bazán, "Transition from bubbling to jetting in a coaxial air-water jet," Phys. Fluids 17, 018105 (2005).

${ }^{34}$ If $L_{b}$ is the length of a bubble then the pressure drop along its length is $\Delta p_{b} \approx\left(p_{\text {gas }}-p_{\text {out }}\right) L_{b} / W$, where $p_{\text {out }}$ is the pressure at the outlet. The relative change in the bubble volume over the length $L_{b}$ will be $p_{\text {gas }} /\left(p_{\text {gas }}\right.$ $\left.-\Delta p_{b}\right)$, using Eq. (2). Compressibility effects are hence pronounced if $\Delta p_{b} / p_{\text {gas }}$ is large; i.e., if $L_{b} / W$ is large or if $p_{\text {gas }}$ and $p_{\text {out }}$ are lowered by the same amount.

${ }^{35} \mathrm{P}$. Garstecki (private communication). 